\title{
Quantum Computing - from NISQ to PISQ
}

\author{
K. Bertels ${ }^{1,2}$, A. Sarkar ${ }^{1,3}$, and I. Ashraf ${ }^{1,4}$ \\ ${ }^{1} Q B e e X$ \\ ${ }^{2}$ University of Leuven, Belgium and University of Porto, Portugal \\ ${ }^{3}$ Delft University of Technology, Netherlands \\ ${ }^{4}$ HITEC University, Pakistan
}

\begin{abstract}
Given the impeding timeline of developing good quality quantum processing units, it is the moment to rethink the approach to advance quantum computing research. Rather than waiting for quantum hardware technologies to mature, we need to start assessing in tandem the impact of the occurrence of quantum computing in various scientific fields. However, to this purpose, we need to use a complementary but quite different approach than proposed by the NISQ vision, which is heavily focused on and burdened by the engineering challenges. That is why we propose and advocate the PISQ-approach: Perfect Intermediate-Scale Quantum computing based on the already known concept of perfect qubits. This will allow researchers to focus much more on the development of new applications by defining the algorithms in terms of perfect qubits and evaluate them on quantum computing simulators that are executed on supercomputers. It is not the long-term solution but will currently allow universities to research on quantum logic and algorithms and companies can already start developing their internal knowhow on quantum solutions.
\end{abstract}

\section{INTRODUCTION}

Quantum computing as a scientific field was launched shortly after a talk that Richard Feynman gave in 1986 to highlight the advantage of simulating quantum dynamics on controllable quantum systems, with respect to classical computers. This inspired the quantum physics community to look at these challenges and realistically start manufacturing these devices. The theoretical and applied benefits of quantum computing algorithms, discovered in the 1990s, established this field as a concrete research direction. Quantum computing got a huge boost from the slowdown of the Moore's law of transistor scaling, resulting in major industrial players investing in the development of scalable systems. The main ideas of our quantum research came out of the collaboration between Intel and our research group in TU Delft. Before we briefly explain part of the full stack, we want to highlight the US-consulting company Gartner's Hype cycle as shown in their well-known Figure that shows the overinvestment in technology in the first cycle, then there is top of the cycle where the deliveries are not really realised and then under-investment will appear. We see that quantum computing is in the first rise of the hype cycle. Universities and companies have to be careful about where to invest in what. This paper will define what the common strategy for this technology in its current state could be.

Soon many different approaches were discovered to make quantum bits, called qubits. Now we are in a phase where there are several of these technologies competing to make a good quality qubit. The longterm goal is to fabricate a quantum chip with a high number of good-quality logical qubits, which can be implemented using quantum error correction.

This is called FTQC standing for Fault-Tolerant Quantum Computing. The next step in quantum computing is now called NISQ which stands for Noisy Intermediate-Scale Quantum computing. the main trend is actually to go back to simpler qubits but trying to solve many of the observed errors. In general, the word 'noisy' refers to the gate errors and decoherence we observe in the physical qubits, the term 'intermediate-scale' refers to the number of physical qubits, such that we cannot exhaustively simulate them on a classical computer. In this paper, we introduce an alternate but closely related concept, called PISQ which stands for perfect intermediate scale quantum computing as will be explained later.

Figure 1 shows the full stack [1], [2] which can be briefly described by the most important layers that are now active. At the highest level, the application is formulated into a quantum algorithm, using the quantum logic primitives from the quantum library. The algorithms describe how many qubits need to be used and what operations should to be performed among them. The application is expressed in a programming language that many companies and universities have 


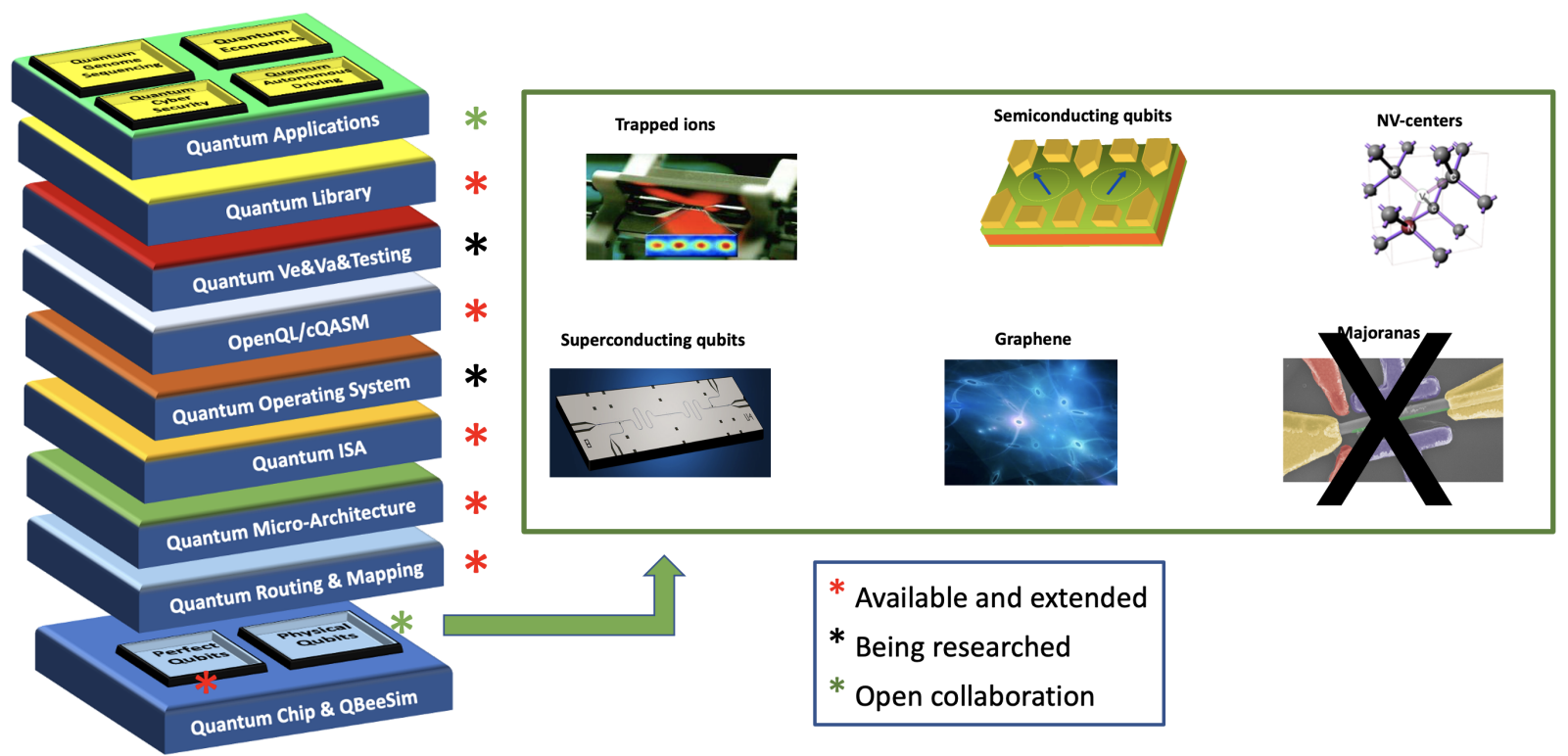

Fig. 1: Full Stack and Qubit technologies

developed. We use the OpenQL language [3] and the compiler translates the OpenQL instructions to cQASM [4], the common quantum assembly language. A micro-architecture receives the CQASM-instructions and does an internal processing before sending it to the lowest level which can be a physical quantum chip or a quantum simulator. The competing technologies are depicted in this figure as well. The highperformance quantum computing simulator [5] we use is developed by our team with a focus on scalability than any other simulator on the market. For every QBeeSim-simulator instance running on a single node, we can go up to 28 qubits in arbitrary superposition. Running multiple QBeeSim-instances at the same time, can substantially increase the number of qubits used in the algorithm.There are simulators around which can simulate up to 64 qubits on a multi-node system [6].

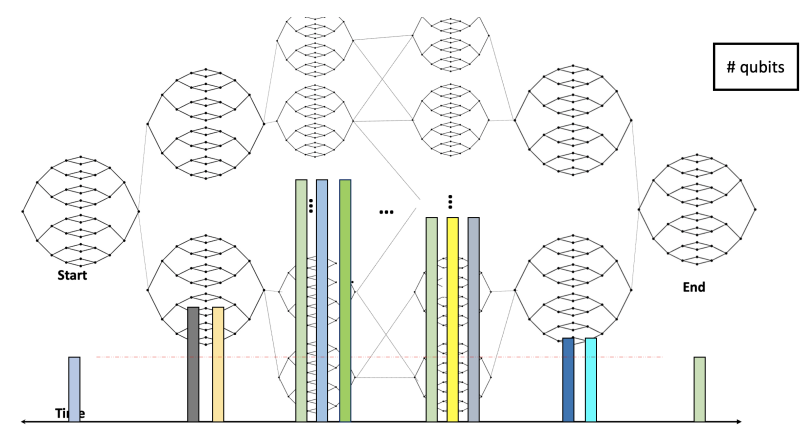

Fig. 2: Number of Qubits grow and shrink during the execution of the quantum application

It is important to understand that real quantum applications will consist of several independent parts. Only the result of the intermediate parts need to be transferred to the next step in the quantum applications where multiple results from different preceding steps are combined. Figure 2 shows this evolution of number of qubits and one can imagine that a single node sends its results to the neighbour following the overall execution of the quantum application. This is the main idea of combining in our case multiple instances of the QBeeSim simulator to achiever greater number of qubits used.

\section{World-wide academic implications}

The quantum physics community is sufficiently aware that if certain quantum technologies do not produce any reasonable result after several years of effort, they should be gently removed from the list of quantum candidates. In the remainder of the paper, we stay very convinced that quantum computing will offer substantial new advances in all scientific fields but we still have a long road ahead. Without going in too much detail, we mention universities and companies as the main drivers of education and research and economic applications. The first main observation to start from is that quantum computing will revolutionise the world as all concepts, theories and applications will have to be redeveloped from scratch as the quantum concepts and corresponding ways to make computations will substantially change. Where in the classical hardware and software it was enough to recompile the same or changed algorithm to have the improved version running on the newest hardware, this is no longer true 
for the quantum technology. We have been working for the last 3-4 years on genomics and we have discovered that the algorithms are completely different than the classical genome sequencing algorithms. Functionally they still do the same thing, for instance, reconstruct the genome based on experimental observations but the way the computations and logic is done has completely changed. We have started looking at chemical applications and also there we see a radical change in the logic expressed in terms of qubits and quantum gates. The main implication of this observation is that anything in science for which we need a computer, needs to be redeveloped and looked at. It does not mean that we do not need classical computers anymore but the size of the problems we can look at will radically change and the kind of operations applied on the qubits will change too. This observation has two main consequences. Every university will have to initiate quantum based research in every scientific field they are working on. This can never be done at a complete university level but every faculty should be invited to encourage young academic members to include quantum computing in their research. Every faculty will need to initiate that process and should hope that more and more faculty members will make the step to quantum research. It takes several years to arrive at a really good level of expertise and become a leader in the scientific field. The second consequence is for companies. Independent of the kind of activity a company is involved in, the influence of quantum computing will also be felt there. Whether the company is a car manufacturer, a bank or a chemistryoriented company, the implications will be clearly felt. Similar to universities, it is not a challenge that needs to be resolved in 3-4 years time but companies should look at it now to start creating a small team of experts that show clear interest in quantum computing. A small team should maybe ask for graduate students to base their thesis on the kinds of problems the company is looking at. Nothing groundbreaking should be expected, but cannot be excluded, but it takes time to have a group inside the organisation capable of looking at the problems the company is looking at. Companies such as Airbus, BMW and others are already looking at those kinds of directions and nobody should wait and see the competition has done it substantially and is bypassing the company.

Before describing in more detail the PISQ-vision, we want to emphasise that the consequences for any scientific and economic field of ever building a quantum computer are simply enormous. When a new classical processor or computer came to the market a mere recompilation of the existing or improved applications was enough to exploit the new hardware features. With a quantum accelerator, part of the research needs to lead to substantial changes to the proposed solutions. In the next pages, we will emphasise what the main physics-oriented challenges are in quantum computing and we will propose an alternative strategy and vision that keeps the research activities open for all kinds of scientific fields. We will describe the kind of research we are currently doing within a company we created but that we are also doing in collaboration with other universities.

\section{Quantum Physics Challenges}

Here we review some of the more important open issues. Our main starting point is a talk John Preskill gave in 2018 about the challenges in quantum computing. In two accompanying papers [7] [8], Preskill targets both active researchers in quantum computing as well as a broader audience. We will highlight the arguments that are relevant for physics researchers.

- Diversity in quantum technology: as shown in Figure 1, many technologies are still competing with each other to make the best qubit. It is uncertain what technology will win. For now, the Majorana approach seems to be falling off as the physics group was not capable of making a single physical Majorana qubit. It is to be expected that many other qubit technologies will disappear in the coming years. We just need to remember that in transistor development, it took the entire world around 40 to 50 years to reach a VLSI-level of transistor production, which is based on an idea formulated in 1936.

- Number of qubits: 50 physical qubits is what Preskill calls a significant milestone as it implies that the physical community is capable of going beyond the capacity of classical computers. The number 50 is motivated by the maximum number of qubits we can simulate when we use modern computers. The main memory in supercomputers stores all the information of the full quantum circuit, implying the basis states and the amplitudes. However, with all the problems qubits still have, it is very doubtful how to interpret and assess the computed results.

- Coherence of qubits: The coherence time is the time the information contained by the qubit is accessible and usable. This coherence time varies significantly depending on the quantum technology used. For different quantum technologies, we have found numbers that go from a couple of seconds to multiple minutes. What is important to realise is that the coherence time needs to be substantially longer than the time it takes to execute the full quantum circuit because otherwise all intermediate or final results will be lost.

- Quality and number of quantum gates: The accuracy of the quantum gates is also a big problem as 
the error rates are way too high to implement any meaningful application that can be tested on its quantum formulation. Preskill suggests to limit it to 1000 gates as the noise will be so high that it is difficult to assess the quantum results obtained. This is certainly meaningful for qubit development but we need to look at many other aspects too to have substantial improvements for any scientific, technological and in general societyrelevant applications.

- Quantum error correction: Given the errors in the computations and the overall behaviour of any qubit technology, there is a need to correct the quantum (intermediate) results such that the qubit states do not incorrectly accumulate all the errors of the preceding computations. These days, the error rates are $10^{-2 /-3}$ and it is interesting to understand what the qubit engineering researchers want to achieve in, for instance, 5 years from now. In CMOS, we are used to having $10^{-15}$ and that is far too ambitious for the next 10 years. But important to know is when can one expect to reach $10^{-6}$, which most likely is still 10 years or longer away.

- Logical qubits: An important attempt was to formulate logical qubits based on multiple physical qubits. The goal is to have an overall qubit behaviour which is more stable and scalable. One approach was based on surface codes but for now we need around 49 physical qubits to have one logical qubit. So, also surface code and other logical-qubit approaches will have to be postponed or substantially reduced in size.

- Scalable fabrication of qubits: It is not yet conceivable to develop fabrication technologies as long as there is no understanding and agreement on what quantum technology can be used to produce good qubits. It is unlikely that all quantum technologies will survive and it will most likely be the role of a small number of big players that will outline what technology will reach the market.

- Quantum oracles: Most of the theoretical research on quantum algorithms proved speedups by looking at a specific part of the overall algorithm. While applying the algorithm in practice, it is needed to holistically consider the oracle as well, the part considered as a black-box in the original formulations. The oracle is a way to verify what a particular function is computing, respecting the reversibility of quantum logic and without knowing what logic the oracle applies.

- Variational heuristics: More recently a lot of focus has been on variational heuristics where a parametric circuit is trained on a quantum computer using a classical optimiser. Algorithms like variational quantum eigensolver (VQE) and quantum approximate optimisation algorithm (QAOA), while at one end promises quantum advantage in the NISQ, there are also many theoretical results that limit their universality.

The NISQ-approach is clearly a very promising direction in which quantum physics can continue researching the development of qubits. The list of challenges that we discussed in this section clearly focuses on a lot of engineering aspects that can be solved by researchers with a quantum physics background.There is also no agreement on how we define the words good and scalable where scalability is needed to compensate for the errors in qubit behaviour as well as other needed elements such as dilution refrigerators and qubit connectivity. So, 50 really good qubits with error rates of $10^{-8}$ are very important for the world. However, as we are now only capable of making 50 qubits with error rates of $10^{-2}$, it is very problematic for the world. That is why we propose an alternative approach where a wider interest community can start looking at the development of quantum solutions and algorithms.

\section{PISQ - PERFECT INTERMEDIATE-SCALE QUANTUM COMPUTING}

Given the full stack as shown in Figure 1, any scientific field should open up to other scientific areas, going beyond the physics dimensions. That is what Figure 3 represents where TRL stands for Technology Readiness Level. Similar to Preskill's message, we have to start working substantially on a wide variety of application domains and expand the number of quantum gates and try to push the number of qubits on supercomputers higher than 50. Just to make the picture complete, we will also use the Tensor mathematics to formulate any quantum operation on any number of combined qubits.[9][10]

In that sense, we are advocating a scientific approach called Perfect Intermediate-Scale Quantum computing, abbreviated to PISQ. The abbreviation is based on the NISQ-vision where the $\mathbf{N}$ refers to noisy intermediate-scale challenges that we discussed in the previous section.

The $\mathbf{P}$ refers to the use of perfect qubits that do not decohere and have perfect quantum gates. Our quantum compiler generates cQASM based on the OpenQL language. When we needed to test the superconducting or semiconducting qubits in the Intel context, we introduced a back-end compiler pass that translates the cQASM version to either the eQASM version for semiconducting or superconducting qubits. Our vision is that we can go to any qubit technology such as photonics or ion-traps in the same way. However, for application and algorithm developers working on the highest layer of the full stack, it is clear that they 


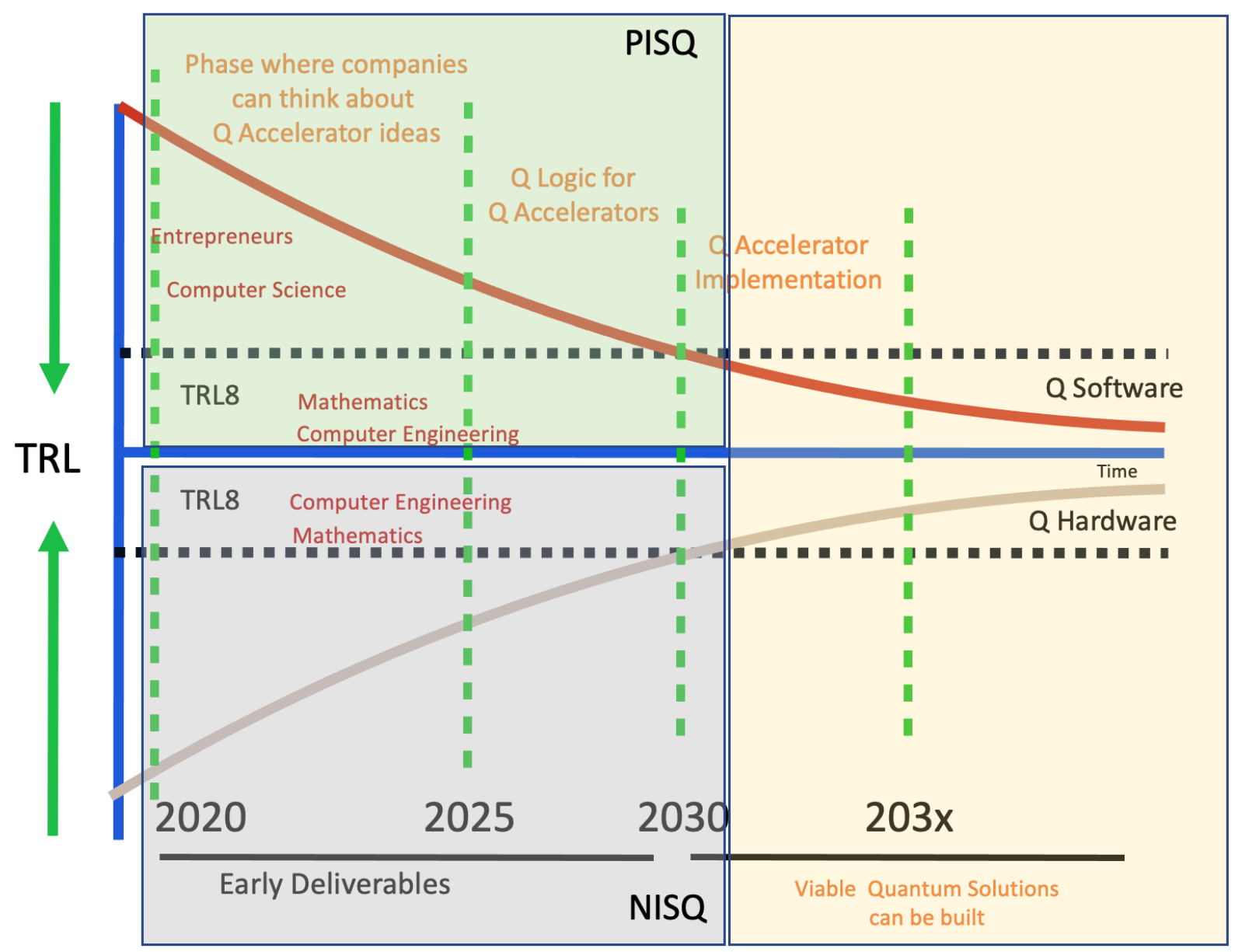

Fig. 3: Complementary PISQ and NISQ quantum research activities

only intend to express their concepts and ideas in terms of perfect qubits and verify the outcome of the computation. The intermediate-scale refers to the number of quantum gates in the circuits which for now goes to 20000 or higher and the number of qubits is still restricted to 50. Important to highlight that most quantum computing companies, like IBM, Rigetti, Google, Microsoft, support quantum development platforms, such as Qiskit, Forest, CirQ and QDK. That includes compilers and simulators capable of generating and executing quantum logic in terms of perfect qubits.

Figure 3 divides the quantum research in three parts. We first have the green and grey periods that start now and run up to 2030. The grey part refers to the NISQ-approach as formulated by Preskill and adopted by the quantum physics community. The green part then refers to the PISQ-approach where it is assumed that the quantum applications are written in perfect qubits. The yellow part on the right of the figure represents the merging of quantum software and hardware in full accelerators and which have been substantially tested. We expect this to happen around 10 years from now but it may be sooner or even beyond 20 years. We now present some of the challenges researchers need to look at when going for the PISQ-approach.

- Quantum Applications: An important topic is the fact that quantum computer devices will change radically all scientific fields as the concepts and problems defined in terms of qubits and quantum gates will require a new generation of scientists to develop the tools and algorithms that will describe in a meaningful and consistent way how problems from Chemistry, Biology, Economics, ... will look like and what kind of solutions they propose.

We just have to realise that the quantity of data available these days is orders of magnitude too big for the current computational power we have. As shown in Figure 4, We have already been looking at quantum genomics and we now start looking at quantum chemistry to understand what 
the impact will be of quantum accelerators on that particular science. This is therefore an open invitation to all university and research groups to start investigating the impact of quantum logic on their problems.

- Parallelisation: There is one concern that cannot be overlooked by the researchers going in the PISQ-direction and that deals with the parallelisation of the quantum algorithm or circuit that will be executed on the classical supercomputer. In the NISQ-approach, the parallelisation is done in an implicit way by the qubits which execute implicitly the possible solutions. When we want to execute a similar circuit on a supercomputer, we need to have an explicit parallel version of the circuit in order to have an equal amount of solutions that the quantum physics execution would generate.

- Supercomputers: Where Preskill talks about 50 qubits running on supercomputers as the upper limit, we will also be confronted with a similar limitation. But because the qubits are perfect, there is no uncertainty with respect to the generated results. Most quantum compilers have a compiler option to generate and simulate a circuit using perfect qubits. It remains to be seen how many more qubits can we superpose to go beyond 50 perfect qubits and execute it on a supercomputer.

- Classical memory use: Any quantum accelerator will have to represent the relevant qubit states after an intermediate step of the quantum application has been completed. This is needed as the quantum simulator such as QBeeSim, needs to be reset and its memories need to be emptied. Intermediate solutions needed for the next step in the quantum application can be stored in the local memory of the quantum accelerator and be transferred to any simulator using it. Such local memory is classical and needs to represent the qubit basis as well as the amplitudes of each state.

- Number of qubits and gates: As discussed in the previous section, a second limitation that the

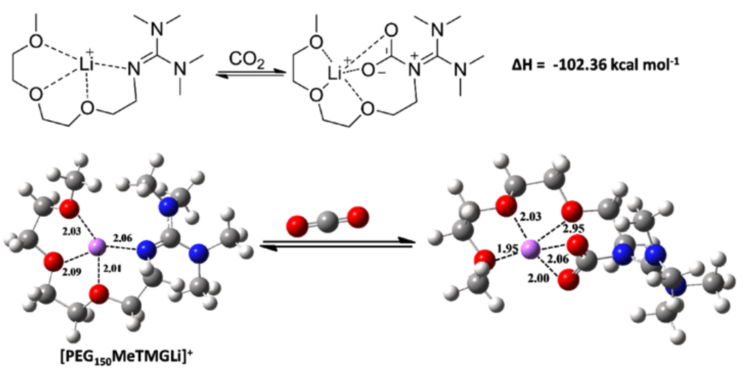

Fig. 4: chemistry
NISQ-researchers need to overcome is the size or length of the quantum circuits. From a physical qubit decoherence point of view, Preskill limited it to 1000 gates but our PISQ-driven research can easily execute circuits with up to 10000 gates and higher in reasonable run time.

We have noticed that the number of gates is not demanding additional memory use of the classical computer so we do not have to limit the size of the circuits to any particular number of gates. The main challenge stays the number of qubits that we can entangle or put in superposition. We are currently investigating how we can increase the number of qubits to get closer to 100 or 150 fully connected qubits using special kind of quantum simulators based on tensor networks and structure within the quantum circuit to be simulated.

- Quantum Random Access Memory: QRAM is a useful primitive to store the classical or quantum data of an algorithm in quantum memory so that it can be accessed on demand. While many quantum algorithms depend on the existence of an efficient QRAM, in practice QRAMs are difficult to build, and the assumption of these algorithms needs to be holistically reviewed and the focus on classical data load needs to be researched. At times, a much benign QROM (quantum read-only memory) suffices.

- Specialised quantum gates: Each qubit technology currently being investigated has their own native gate set that supports universal computing by translating other logic to a decomposition of the native gate set. These translations implies that some applications are much easier to translate for one qubit technology than other based on how easy it is to decompose the most used logic in that application algorithm for the qubit technology. In this view, it might be useful to develop quantum hardware that are specifically designed to support specific applications, e.g. specific controlled rotations for QFT.

In terms of applications, it is clear that the physics field is very inquisitive to explore complicated problems from their field. However, there is in principle no limitation to any topic for the quantum application layer. We personally work on quantum genomics [11] and quantum finance, but topics coming from chemistry, biology and any other field are also fashionable. The PISQ-approach will allow universities to start developing research in any scientific field as the impact of QC on any scientific field will be incredible. It is advisable to start that new line of research perspective as soon as possible. It will take a new generation of scientists to study their core problems and find quantum algorithms that will solve that problem. It is evident that given the current state of supercomputers and 
qubits, the problems have to be reduced substantially that it can be executed by quantum simulators. This is the main reason to adopt the PISQ-approach for young academic people as well as for dynamic entrepreneurs to start reasoning about their hard problems already now.

\section{Conclusion}

In this relatively short paper, we propose the PISQoriented research line such that more people from any scientific field will step towards research for quantum computing. In our multiple years of past collaboration in Delft, the quantum computer architecture team focused on both semiconducting and superconducting qubits. We developed our OpenQL programming language [3] such that it could be translated to cQASM [4] and later to an eQASM [12] version which could control either of the two or more qubit technologies. The same approach is now continued much more explicitly using the already existing notion of perfect qubits. While most quantum computing frameworks also provide this as an alternative, the perfect simulator is typically not advocated in the final product development to encourage more widespread adoption of the NISQ-era platforms. In this article, we pointed out the pitfalls of using this for product development road-maps.

Quantum applications formulated using perfect qubits can be executed and tested on classical QC simulators. This way, we can study and analyse new quantum algorithms for any kind of complex problems, that we cannot address even with supercomputers and classical programming. The constraint is that the number of qubits stays relatively low but one has a scalable formulation of the quantum solution, which can be immediately targeted to a quantum chip when they reach technological maturity.

To provide this important direction of research and development with an identifiable banner we introduce the notion of PISQ, which stands for Perfect Intermediate-Scale Quantum computing. It is a complementary approach to NISQ, to work on quantum computing and, as stated, it may have a substantial impact on all the scientific and even economic efforts world-wide. The main advantage is that there is no direct dependence on the roadblocks and progress of the quantum physical chip development efforts. In the long term, Fault-Tolerant Quantum Computing will allow us to integrate NISQ and PISQ results. That means that application developers do not have to worry about decoherence and quantum errors in the operations but rather focus on the quantum logic for important problems of their interest.

\section{REFERENCES}

[1] K. Bertels, A. Sarkar, T. Hubregtsen., M. Serrao, A. A. Mouedenne, A. Yadav, A. Krol, and I. Ashraf. Quantum computer architecture: Towards full-stack quantum accelerators. In 2020 DATE, pages 1-6, 2020.

[2] K. Bertels, A. Sarkar, A. Krol, R. Budhrani, J. Samadi, E. Geoffroy, J. Matos, R. Abreu, G. Gielen, and I. Ashraf. Quantum accelerator stack: A research roadmap. arXiv 2102.02035, 2021.

[3] N. Khammassi et al. Openql: A portable quantum programming framework for quantum accelerators. arXiv preprint arXiv:2005.13283, 2020.

[4] N. Khammassi et al. cqasm v1. 0: Towards a common quantum assembly language. arXiv preprint arXiv:1805.09607, 2018.

[5] Ravish Budhrani. Quantumsim: A memory efficient simulator for quantum computing. Master's thesis, Delft University of Technology, 2020.

[6] Zhao-Yun Chen, Qi Zhou, Cheng Xue, Xia Yang, GuangCan Guo, and Guo-Ping Guo. 64-qubit quantum circuit simulation. Science Bulletin, 63(15):964-971, Aug 2018.

[7] John Preskill. Quantum Computing in the NISQ era and beyond. Quantum, 2:79, August 2018.

[8] John Preskill. Quantum computing 40 years later, 2021.

[9] E. Desurvire. Classical and Quantum Information Theory. Cambridge University Press, 2009.

[10] Tensor_wikipedia. Tensor mathematics. https://en. wikipedia.org/wiki/Tensor

[11] Aritra Sarkar. Quantum algorithms for pattern-matching in genomic sequences. Master's thesis, Delft University of Technology, June 2018.

[12] Xiang Fu et al. eqasm: An executable quantum instruction set architecture. In 2019 IEEE International Symposium on High Performance Computer Architecture (HPCA), pages 224237. IEEE, 2019.

\section{Authors}

Koen Bertels is part-time and for 3 years Professor in Quantum Computer Architecture at the University of Leuven and at the University of Porto. He works on computer engineering aspects of realizing a quantum accelerator investigating the challenges of system design and architecture. The focus is on scaling challenges, investigating how a large number of qubits can be efficiently used and controlled. His group works on all the layers of the full computer stack. For application development, he emphasizes the use of perfect qubits, without decoherence and gate errors. He is excited about quantum computing as the ultimate accelerator giving access to problem domains that are out of scope in conventional computing. Contact him at koen.bertels@qbee.com.

Aritra Sarkar is a Ph.D. candidate at QuTech, in the department of Quantum and Computer Engineering at the Delft University of Technology. He received his Bachelor of Technology (B.Tech), in Avionics, from the Indian Institute of Space Science and Technology, Thiruvananthapuram, India, in 2013. He worked in the Indian Space Research Organisation as a Scientist till 2016. He completed his Master of Science (M.Sc), in Computer Engineering, from the Delft University of 
Technology, Delft, Netherlands, in 2018 and then joined the Faculty of Electrical Engineering, Mathematics and Computer Sciences, towards a Doctor of Philosophy (Ph.D.) in quantum algorithms. His research interests lie in quantum acceleration for experimental algorithmic information theory applications in artificial general intelligence and genomics.

Imran Ashraf received his Ph.D. in Computer Engineering from Delft University of Technology, The Netherlands in 2016. The focus of his research was advanced profiling, accelerator-based computing, communication driven mapping of applications on multicore platforms. In 2016, Imran started working as Post-Doctoral Researcher on Intel funded project at Quantum and Computer Engineering department, QuTech, TU Delft. His research focused on simulation and compilation techniques for quantum computing and scalable architectures for quantum computers. Currently, Imran is working as an Assistant Professor at Computer Engineering Department, HITEC University, Taxila, Pakistan. 